〔Med. Entomol. Zool. Vol. 54 No. 1 p. 37-42 2003]

\title{
A comparison of improvemental ability of water quality among five chironomid species of the genus Chironomus
}

\author{
Koichiro KawaI ${ }^{1)}$, Toshiko Kawai ${ }^{2)}$ and Hiromichi Imabayashi ${ }^{1)}$ \\ 1) Laboratory of Ecology, Faculty of Applied Biological Science, Hiroshima University, \\ Kagamiyama 1-4-4, Higashihiroshima, Hiroshima, 739-8528 Japan \\ 2) Shirokane Post Office, Nishiyoshino-mura 536, Yoshino-gun, Nara, 639-1102 Japan
}

(Received: 7 March 2002; Accepted: 20 November 2002)

\begin{abstract}
Five chironomid species of the genus Chironomus were examined for caloric contents in adults and for their ability of calory removal from bodies of water by emergence and of water quality improvement in laboratory experiments. Calory per $\mathrm{g}$ adults was the highest (about 5,000 cal) for Chironomus kiiensis, higher than 2,500 cal for C. circumdatus, C. nippodorsalis and C. yoshimatsui, and the lowest (about $2,000 \mathrm{cal}$ ) for $C$. nipponensis. The actual rate of removal by emergence was the highest (about 13\%) for C. nippodorsalis, moderate (about 10\%) for C. circumdatus and C. yoshimatsui, and the lowest (about 6\%) for C. kiiensis and C. nipponensis. For inhibitory ability of eutrophication, in terms of $\mathrm{PO}_{4}-\mathrm{P}$ concentration, about $89 \%$ of inhibition was observed for C. nipponensis. Inhibition was higher than $70 \%$ for $C$. circumdatus and C. yoshimatsui, about $31 \%$ for C. nippodorsalis, and the lowest (about $24 \%$ ) for C. kiiensis at 4 weeks. For inhibitory ability in terms of protein concentration, inhibition was the highest (about 80\%) for C. circumdatus, higher than $50 \%$ for $C$. kiiensis, C. nipponensis and C. yoshimatsui, and the lowest (about 41\%) for C. nippodorsalis at 4 weeks.
\end{abstract}

Key words: Chironomidae, improvement, rearing experiment, water quality

\section{INTRODUCTION}

Chironomid larvae live in a wide variety of inland waters in high density, feed actively on algae, detritus or other benthic invertebrates, grow rapidly, metamorphose to adults, and emerge into the air in large masses. Therefore, they are considered to play an important role in the circulation of materials and in suppression of eutrophication in aquatic ecosystems. Particularly, the genus Chironomus whose larvae are abundant in more or less eutrophicated waters (Sasa and Kikuchi, 1995), is considered to be the most important genus in this respect. Iwakuma (1990) has demonstrated a suppression of regression of nitrogen from sediment to water by the larval development of two large profundal species, Chironomus plumosus and Propsilocerus akamusi in Lake Kasumigaura, Japan. Larvae of C. circumdatus have been reported to remove daily up to $31 \%$ of the organic matter in a sewage canal (Sankerperumal and Pandian, 1992). In our previous study, five Japanese species of the genus Polypedilum were examined for their ability to remove organic materials from bodies of water by measuring caloric contents of emerging adult midges and for their ability in improvement of water quality by monitoring concentrations of $\mathrm{PO}_{4}$-P and protein in waters, and $P$. sordens was considered to have the highest ability in improving water quality (Kawai et al., 2000). However, no studies have been conducted on the differences in the ability to remove organic materials and to improve water quality among various species of 
the genus Chironomus under experimental conditions.

In this study, five Japanese species of the genus Chironomus were selected as materials for experiments, and they were examined to determine their ability to remove organic materials and to improve water quality by the same methods as the previous study.

\section{Materials And Methods}

\section{Chironomids}

Larvae of Chironomus circumdatus, C. kiiensis, C. nippodorsalis, C. nipponensis and $C$. yoshimastui were obtained by laboratory culture. The conditions for obtaining fertilized egg masses and rearing of the larvae were described in Kawai and Konishi (1986) and others.

\section{Rearing method}

The newly hatched larvae were reared at a density of 200 individuals per container. Each container $(\phi 15 \times \mathrm{H} 9 \mathrm{~cm})$ was layered with $150 \mathrm{ml}$ of $1 \%(\mathrm{w} / \mathrm{v})$ agar plate containing $2 \%(\mathrm{v} / \mathrm{v})$ milk (fat content $3.5 \%$ ) on the bottom, filled with 500 $\mathrm{m} l$ of dechlorinated water, aerated through an airstone at room temperature and covered with a nylon net on the top. A container was set for each species. The water level in the container was kept constant during the experiment by replacing the amount of water lost by evaporation. Emerging adults were collected daily for 4 weeks and preserved as dry specimens. Control containers without larvae were also prepared and kept under the same conditions.

\section{Measurement of calory}

The caloric content retained by dried specimens was measured with a calorimeter (Ogawa Seiki Co. Ltd., Tokyo, Japan) according to the manufacturer's instruction.

\section{Measurement of removal rate of calory}

The caloric removal rate was calculated by the following formula:

Removal rate $(\%)=$ the total calory of emerging adults/the total calory of milk $(1,969 \mathrm{cal})$ put into a container $\times 100$

\section{Measurement of $\mathrm{PO}_{4}-\mathrm{P}$ and protein concen- trations}

A water sample (about $50 \mathrm{ml}$ ) was collected weekly from each container after being gently mixed and settled down. $\mathrm{PO}_{4}-\mathrm{P}$ concentration was measured by the molybdenum blue method as an indicator of concentration of nutritional salts. Protein concentration was measured by the method described by Lowry et al. (1951) as an indicator of concentration of dissolved organic materials.

\section{Measurement of inhibition of eutrophication}

Inhibition of eutrophication was measured by the following formula:

Inhibition $(\%)=\left[\mathrm{PO}_{4}-\mathrm{P}\right.$ (or protein) conc. for control water $-\mathrm{PO}_{4}-\mathrm{P}$ (or protein) conc. for sample water] $/ \mathrm{PO}_{4}-\mathrm{P}$ (or protein) conc. for control water.

\section{RESUlts}

\section{Emergence rate}

The emergence rate was below 60\% for all species (Table 1). It was higher than $50 \%$ for C. circumdatus, C. nippodorsalis and C. yoshimatsui, and was 35-40\% for $C$. kiiensis and C. nipponensis. Only a few larvae or pupae were observed to be alive after 4 weeks, the end of the experiment (data not shown).

\section{Calory content of adult midges}

The total calory of the emerging adults was the highest $(245 \mathrm{cal})$ for C. nippodorsalis. It was in the range of $170-200 \mathrm{cal}$ for C. circumdatus and C. yoshimatsui, and was very low (about $110 \mathrm{cal}$ ) for $C$. kiiensis and C. nipponensis (Table 1).

Calory per g adults was by far the highest (about 5,000 cal) for C. kiiensis. It was higher than 2,500 cal for $C$. circumdatus, $C$. nippodorsalis and C. yoshimatsui, and was very low (about 2,000 cal) for C. nipponen- 
Table 1. Emergence rate and adult calory for 5 Chironomus species.

\begin{tabular}{lcccc}
\hline \hline \multicolumn{1}{c}{ Species } & $\begin{array}{c}\text { Emergence } \\
\text { rate }(\%)\end{array}$ & $\begin{array}{c}\text { Total calory of } \\
\text { emerging adults }\end{array}$ & $\begin{array}{c}\text { Calory per } \\
\text { g adults }\end{array}$ & $\begin{array}{c}\text { Calory per } \\
\text { individual }\end{array}$ \\
\hline Chironomus circumdatus & 58.5 & 176.69 & 2,561 & 1.51 \\
C. kiiensis & 34.5 & 111.39 & 4,973 & 1.61 \\
C. nippodorsalis & 57.0 & 244.77 & 3,335 & 2.15 \\
C. nipponensis & 40.0 & 107.88 & 2,035 & 1.35 \\
C. yoshimatsui & 58.5 & 196.61 & 2,883 & 1.68 \\
\hline
\end{tabular}

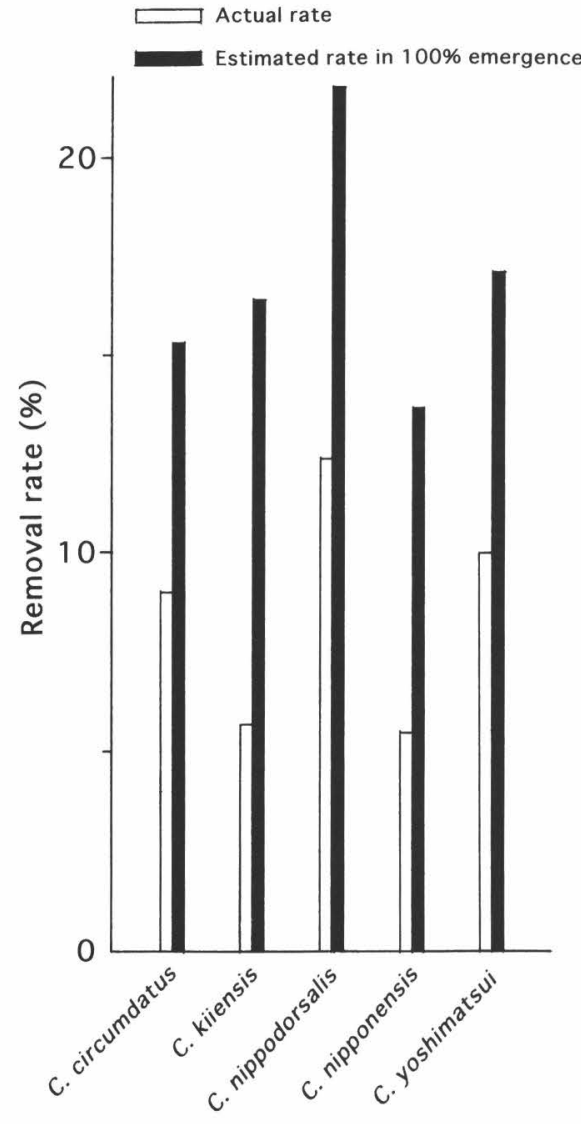

Fig. 1. Differences in removal rates of calory by emergence among 5 Chironomus species.

sis.

Calory per individual was by far the highest $(2.15 \mathrm{cal})$ for $C$. nippodorsalis. It was higher than $1.5 \mathrm{cal}$ for C. circumdatus, C. kiiensis and C. yoshimatsui, and was the lowest $(1.35 \mathrm{cal})$ for $C$. nipponensis.

\section{Removal rate}

The actual rate of caloric removal by emergence was the highest (about 13\%)

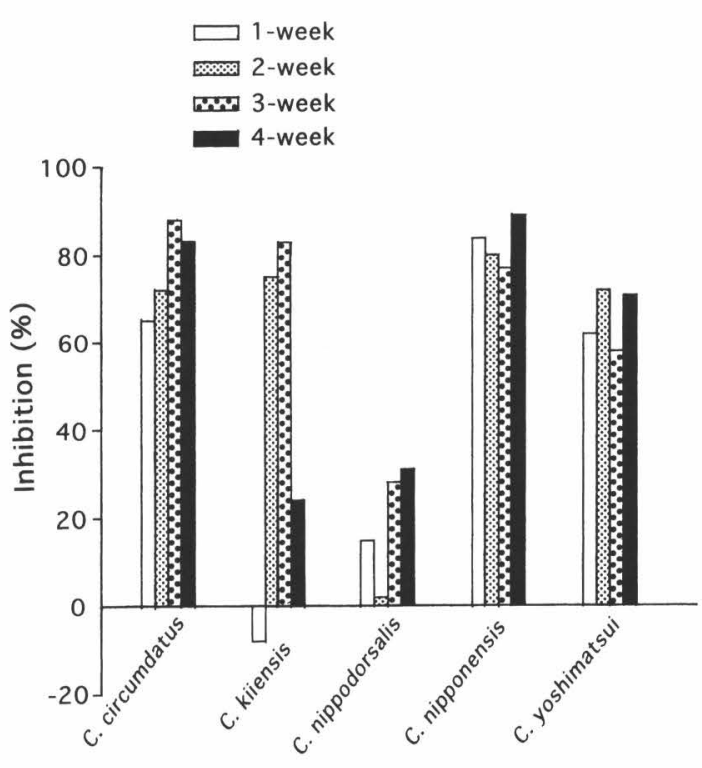

Fig. 2. Differences in inhibitory abilities of eutrophication among 5 species in terms of $\mathrm{PO}_{4}$-P concentration.

for C. nippodorsalis, moderate (about 10\%) for $C$. circumdatus and C. yoshimatsui, and was lower than 6\% for $C$. kiiensis and $C$. nipponensis. The estimated rate of removal in 100\% emergence was the highest (about 22\%) for C. nippodorsalis and was higher than $15 \%$ for $C$. circumdatus, $C$. kiiensis and $C$. yoshimatsui. It was the lowest (about 14\%) for C. nipponensis (Fig. 1).

\section{Inhibition of eutrophication}

\section{1. $\mathrm{PO}_{4}-\mathrm{P}$ concentration}

$\mathrm{PO}_{4}-\mathrm{P}$ concentration of the control water was $0.38,0.72,0.71$ and $0.73 \mu \mathrm{g} / \mathrm{ml}$ at weeks 1, 2, 3 and 4, respectively (Fig. 2). The inhibition rate of eutrophication compared to the control water gradually in- 
creased until the 3 rd week for $C$. circumdatus and $C$. kiiensis. After 1 week, the highest inhibition (about 84\%) was observed for C. nipponensis. Inhibition was higher than $60 \%$ for $C$. circumdatus and $C$. yoshimatsui. Only 15\% of inhibition was observed for C. nippodorsalis. About 8\% of promotion in eutrophication was even observed for C. kiiensis. After 2 weeks, the highest inhibition (about 80\%) was observed for C. nipponensis. Inhibition was higher than $70 \%$ for $C$. circumdatus, $C$. kiiensis and C. yoshimatsui. Practically no inhibition was observed for C. nippodorsalis. After 3 weeks, the highest inhibition (about 88\%) was observed for C. circumdatus. Inhibition was higher than $75 \%$ for C. kiiensis and C. nipponensis. Inhibition was lower than $60 \%$ for C. yoshimatsui. The lowest inhibition (about 28\%) was observed for C. nippodorsalis. After 4 weeks, the highest inhibition (about 89\%) was observed for C. nipponensis. Inhibition was higher than $70 \%$ for C. circumdatus and C. yoshimatsui. Only $31 \%$ of inhibition was observed for C. nippodorsalis. The lowest inhibition (about 24\%) was observed for $C$. kiiensis.

\section{Protein concentration}

Protein concentration of the control water was $85,60,65$ and $81 \mu \mathrm{g} / \mathrm{ml}$ at weeks 1, 2, 3 and 4, respectively (Fig. 3). Inhibition rate compared to the control water gradually increased until the 3rd or 4 th week for all species. After 1 week, inhibition was $12 \%$ at the highest. More than $60 \%$ of promotion in eutrophication was even observed for $C$. circumdatus and C. kiiensis. After 2 weeks, about $50 \%$ of inhibition was observed for $C$. kiiensis and C. nipponensis. Inhibition was higher than $30 \%$ for the other 3 species. After 3 weeks, about $60 \%$ of inhibition was observed for C. circumdatus, C. kiiensis and C. nipponensis. Inhibition was lower than 40\% for C. nippodorsalis and C. yoshimatsui. After 4 weeks, by far the highest inhibition (about 80\%) was observed for C. circumdatus. Inhibition was higher than 50\% for C. kiiensis, C. nipponensis

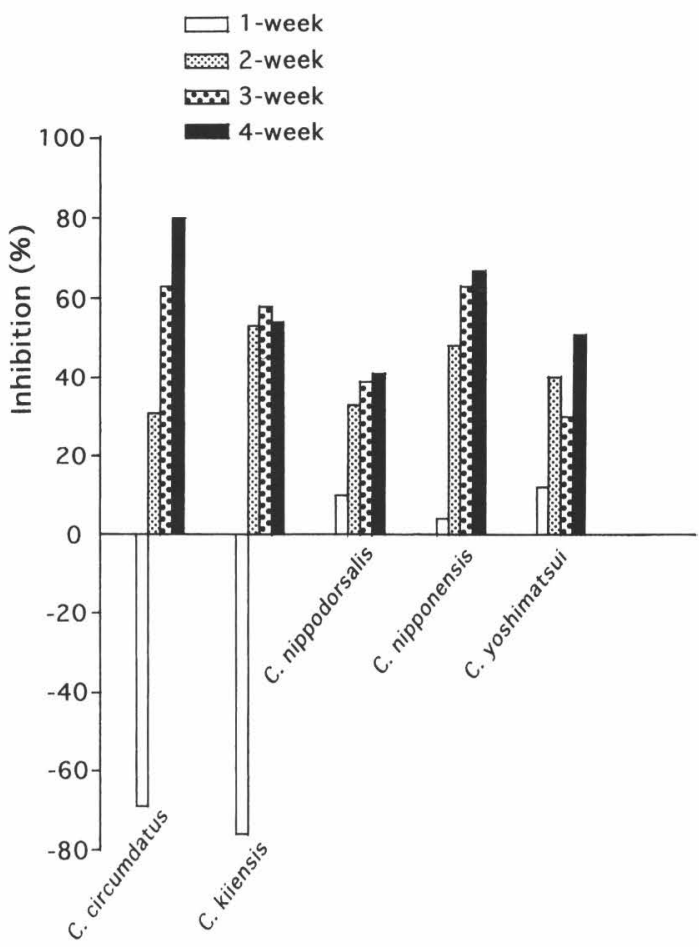

Fig. 3. Differences in inhibitory abilities of eutrophication among 5 species in terms of protein concentration.

and C. yoshimatsui. The lowest inhibition (about 41\%) was observed for C. nippodorsalis.

\section{Discussion}

The emergence rate was lower than $60 \%$ for all the species of the genus Chironomus examined in this study. In contrast, it was higher than $80 \%$ for 4 out of 5 species of the genus Polypedilum examined in the previous study (Kawai et al., 2000). The reason for this is unknown. This might be explained by a stronger density effect (i.e., smaller amounts of various resources available to an individual) exerted on the former than on the latter, resulting from the much larger body size of the former (Wiederholm, 1989). The low emergence rates in the present study are also attributable to the deficiency of granular material for tube-building that is essential to larval life of Chironomus, espe- 
cially for pupation. Indeed, in our preliminary study, a remarkable improvement in emergence rate was attained by the use of agar plates which were spread with fine glass beads on the surface.

On the other hand, a low emergence rate means the death of many larvae during the experiment. Neither the dead larvae were removed nor the water in the container was renewed throughout the experimental period in this study. Therefore, there must have been a considerable level of eutrophication resulting from bacterial degradation of the dead larvae. Nevertheless, remarkable inhibition of eutrophication was observed in the experimental container. Thus, inhibition rates might have been much higher if the emergence rate was rendered $100 \%$.

The calory per gram of adults was in the range of about 2,000-3,300 cal except for C. kiiensis in the present study whereas it was in the range of about $4,700-7,800 \mathrm{cal}$ except for $P$. sordens, in the previous study (Kawai et al., 2000). This may be attributable to the difference in larval foods between these genera. That is, the species of Chironomus are usually scavengers (collector-gatherers) (Beck, 1977), then ingesting low calory, half-degraded materials such as detritus in the experimental container whereas those of Polypedilum are collector-filterers or shredders (Berg, 1995), mainly ingesting high calory, newly produced algae.

In this study, remarkable eutrophication of water was observed early for the control container whereas eutrophication was distinctly inhibited in the experimental container with larvae. This may be explained as follows. In the control container, nutritional materials such as salts and proteins are gradually released from the agar plate and incorporated into bacteria or algae. Bacteria and algae rapidly increase and die, and their dead bodies form a pile of detritus, from which nutrients are again rapidly eluted into the water. In contrast, in a container with larvae, nutrient-incorporating bacteria or algae are rapidly and efficiently ingested and exhausted by the larvae. Thus, detrital compilation is effectively inhibited, resulting in inhibition of eutrophication. That is, there may be some differences in the process and rate of material circulation between the control and experimental containers.

More than $60 \%$ of promotion of eutrophication in terms of protein concentration was observed after 1 week for C. circumdatus and $C$. kiiensis. In our previous study, about $20 \%$ of promotion of eutrophication was also observed after 2 weeks for Polypedilum nubeculosum (Kawai et al., 2000). Use of the interior as well as the surface of the agar plate as a habitat by these species, observed throughout the experiment, might have made the dissolved proteins efficiently elute from the whole agar plate, resulting in such promotion.

Milk-containing agar plates were used for larval rearing from the 1st instar in this study. This plate was worked out in order to strictly examine the differences in energy removal and in inhibition of eutrophication among the species under identical rearing conditions. Considering some differences in major habitats among the species of Chironomus (Sasa et al., 1980; Sasa and Hasegawa, 1983; Yasuno et al., 1983; Kikuchi et al., 1985), however, the results obtained in this study may not necessarily reflect the above-mentioned properties of each species living in the field. Therefore, a detailed study should be conducted on the differences in composition of food materials in the field among the species.

Concentrations of $\mathrm{PO}_{4}-\mathrm{P}$ and protein in the water were measured as an indicator of eutrophication in this study since such measurements neither need a long time, high cost nor skillful technique. However, these items of water quality do not necessarily reflect the trophic status of the water correctly. Therefore, other items, widely used as an indicator, such as $\mathrm{NO}_{3}-\mathrm{N}, \mathrm{BOD}$ and chlorophyll a, should also be measured. 


\section{REFERENCES}

Beck, M. W. 1977. Environmental Requirements and Pollution Tolerance of Common Freshwater Chironomidae. 261 pp., Environmental Monitoring and Support Laboratory, U. S. Environmental Protection Agency, Cincinnati.

Berg, M. B. 1995. 7. Larval food and feeding behavior. In: The Chironomidae (ed. Armitage, P. D., Cranston, P. S. and Pinder, L.C. V.). pp. 136-168. Chapman \& Hall, Great Britain.

Iwakuma, T. 1990. Impacts of benthic macroinvertebrates on the water quality in a basin for municipal water supply. Kogai to Taisaku, 26: 10-16 (In Japanese).

Kawai, K., Kawai, T. and Imabayashi, H. 2000. A comparison of improvemental abilities of the water environment among some chironomid species of the genus Polypedilum. Med. Entomol. Zool., 51: 87-93.

Kawai, K. and Konishi, K. 1986. Fundamental studies on chironomid allergy. I. Culture methods of some Japanese chironomids (Chironomidae, Diptera). Jpn. J. Sanit. Zool., 37: 47-57.

Kikuchi, M., Kikuchi, T., Okubo, S. and Sasa, M. 1985. Observation on the seasonal prevalence of chironomid midges and mosquitoes by light traps set in a rice paddy area in Tokushima. Jpn. J. Sanit. Zool., 36: $333-342$.
Lowry, O. H., Rosenbrough, N. J., Farr, A. L. and Randall, R. L. 1951. Protein measurement with the Folin phenol reagent. J. Biol. Chem., 193: 265-275.

Sankerperumal, G. and Pandian, T. J. 1992. Larval abundance of Chironomus circumdatus in relation to biotic and abiotic factors. Hydrobiologia, 246: 205-212.

Sasa, M. and Hasegawa, H. 1983. Chironomid midges of the tribe Chironomini collected from sewage ditches, eutrophicated ponds, and some clean streams in the Ryukyu Islands, southern Japan. Jpn. J. Sanit. Zool., 34: 305-341.

Sasa, M. and Kikuchi, M. 1995. Chironomidae [Diptera] of Japan. 333 pp., University of Tokyo Press, Tokyo.

Sasa, M., Yasuno, M., Ito, M. and Kikuchi, T. 1980. Studies on chironomid midges of the Tama River. Part 1. The distribution of chironomid species in a tributary in relation to the degree of pollution with sewage water. Res. Rep. Natl. Inst. Environ. Stud., 13: $1-8$.

Wiederholm, T. E. 1989. Chironomidae of the Holarctic region. Keys and diagnoses. Part 3-Adult males. Entomol. Scand. Suppl., 34: 1-532.

Yasuno, M., Iwakuma, T., Sugaya, Y. and Sasa, M. 1983. Benthos in Japanese lakes at different trophic levels. In: Chironomids as Indicator Organisms of Environment, B182-R12-17 (ed., Yasuno, M.), pp. 21-48, Ministry of Education, Science and Culture of Japan, Tokyo (In Japanese). 